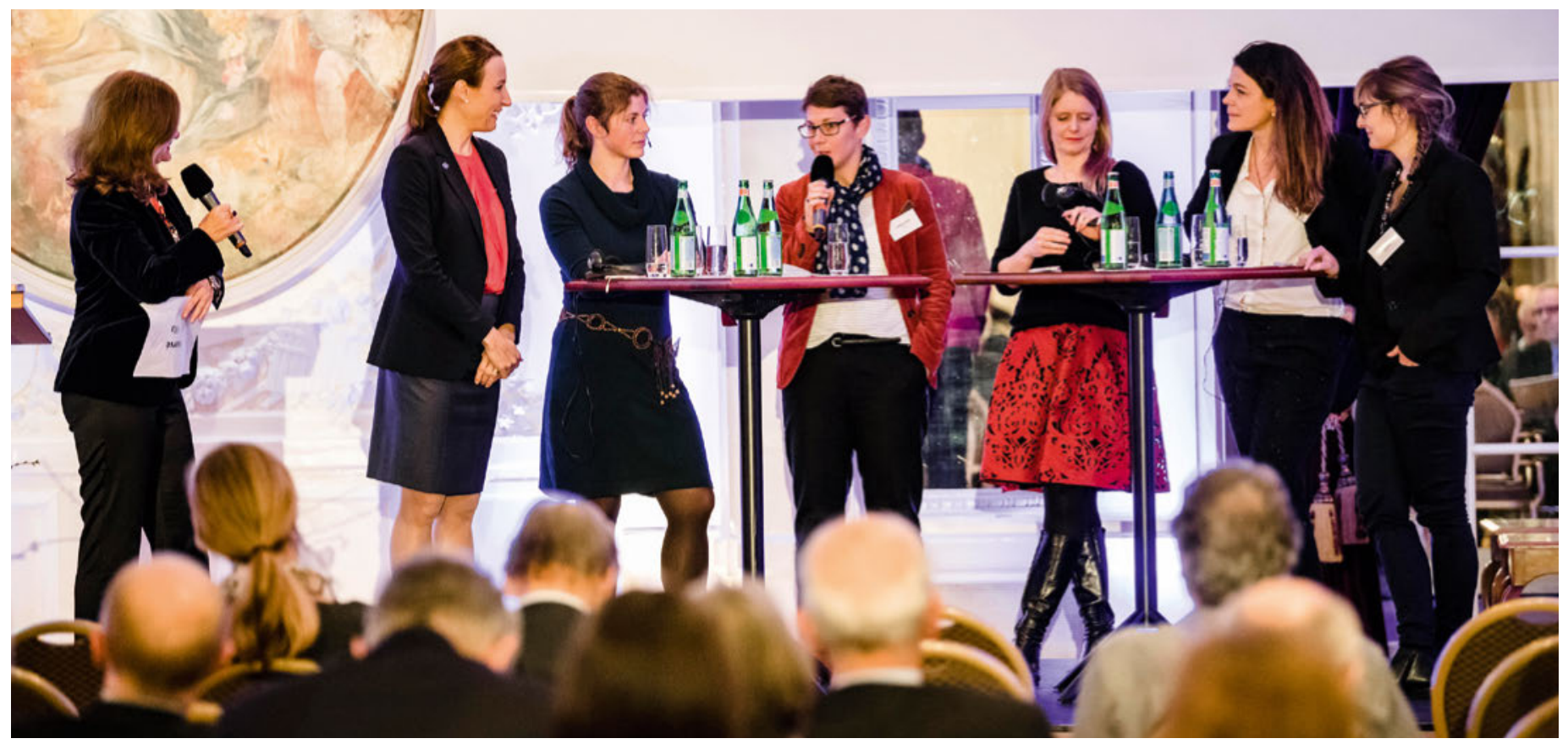

Diskussionsrunde mit sieben Frauen (von rechts): Cornelia Meier, Carole Clair, Solange Peters, Esther Kraft, Gabriela Rohrer, Heidi Stensmyren und Marina Villa (Moderation)

\title{
Zwischen Blindheit und Stereotypisierung
}

\section{Daniel Lüthi}

Freier Journalist und Fotograf, Medientrainer, Bern

«Frauen erobern das Gesundheitswesen - was hat man(n) zu erwarten?»: Dies der Titel einer Veranstaltung, an der kaum Überraschendes zum Thema zu erfahren war. Trotzdem lohnte es sich, den Referentinnen mit offenen Ohren zu begegnen vieles, was sie sagten, gilt es immer und immer wieder zu betonen. Besonders eindrücklich war das Aufeinandertreffen von zwei völlig verschiedenen Schweizer Ärztinnen.

Da war die 46-jährige, auch international erfolgreiche und etablierte Onkologieprofessorin Solange Peters aus Lausanne - und da war die 34-jährige Allgemeinmedizinerin Gabriela Rohrer aus Flühli-Sörenberg, die dort eben eine eigene Hausarztpraxis eröffnet hat. Sie habe keine Statistiken zu bieten, sagte sie entschuldigend und gleichzeitig wohltuend zu Beginn, dann erzählte sie ihre ganz persönliche, berührende Lebensgeschichte.

\section{Ein authentisches Zeugnis}

«Wenn sie wissen, wie es ist, mit beiden Füssen im Mist zu stehen, dann ist es einfach schwierig mit der Sterilität im Spital», setzte sie beispielsweise als Statement in den Saal. Oder: «Es hilft in der Chirurgie, wenn man schon mal kleine Ferkel kastriert hat.» Rohrer hatte in der Landwirtschaft und auf dem Bau gearbeitet, bevor sie Medizin studierte, und dieses Studium und das, 
was danach kam, liess ihre Beziehung zerbrechen und löste eine schwere Depression aus. In ihrer Doktorarbeit setzte sie sich dann mit ihrer Rolle als Ärztin auseinander. Gelernt habe sie dies, hält Gabriela Rohrer fest: «Ich orientiere meine Arbeit an meinen Kräften - und nicht umgekehrt. Und: Ich vertrete meine Standpunkte.» Nötig seien aber nicht nur eigene Erkenntnisse, sondern auch Hilfestellungen von aussen: ein Mann, der auch mal zurückstecken könne, Vorbilder, Förderer und Kraftquellen.

\section{Bewusstsein ist wichtig}

Vor einem ganz anderen Hintergrund und in einem ganz anderen, nämlich rasenden Tempo referierte Solange Peters, Onkologie-Professorin und Führungsmitglied am CHUV in Lausanne sowie Präsidentin der «European Society for Medical Oncology ESMO» mit rund 40000 Mitgliedern. Wenn eine Frau in der Medizin Karriere machen wolle, müsse sie zuerst Mentoren identifizieren, dann Sponsoren und Fürsprecher, sagte sie. Peters beklagte den niederen Prozentsatz von Frauen in Führungsgremien und die grossen Unterschiede bei den Salären, sie vermied persönliche Bezüge, zeigte zahlreiche Folien und Statistiken und fasste ihr Plädoyer mit dem Satz zusammen: «Änderungen sind politisch schwierig zu erreichen - aber die Betrachtungsweise kann sich ändern.» Dabei sei nicht das eigene Verhalten der Frauen entscheidend, sondern die Art, wie sie behandelt würden.

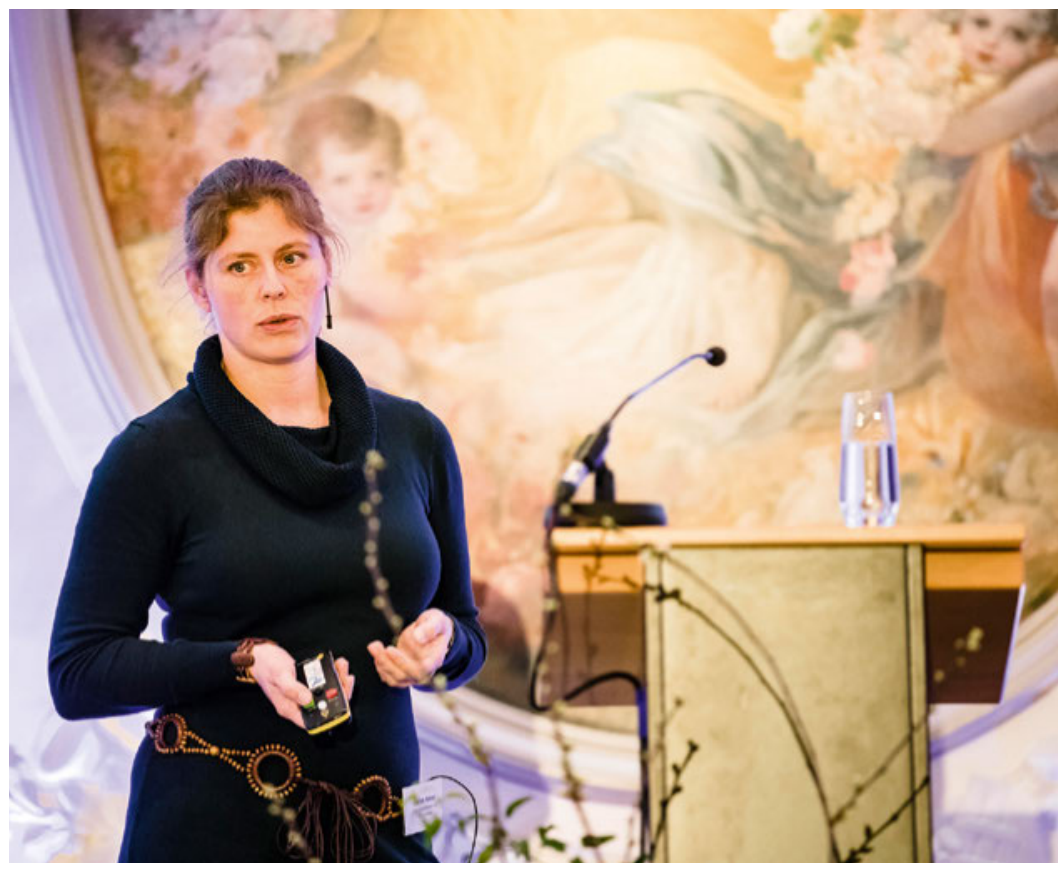

Gabriela Rohrer

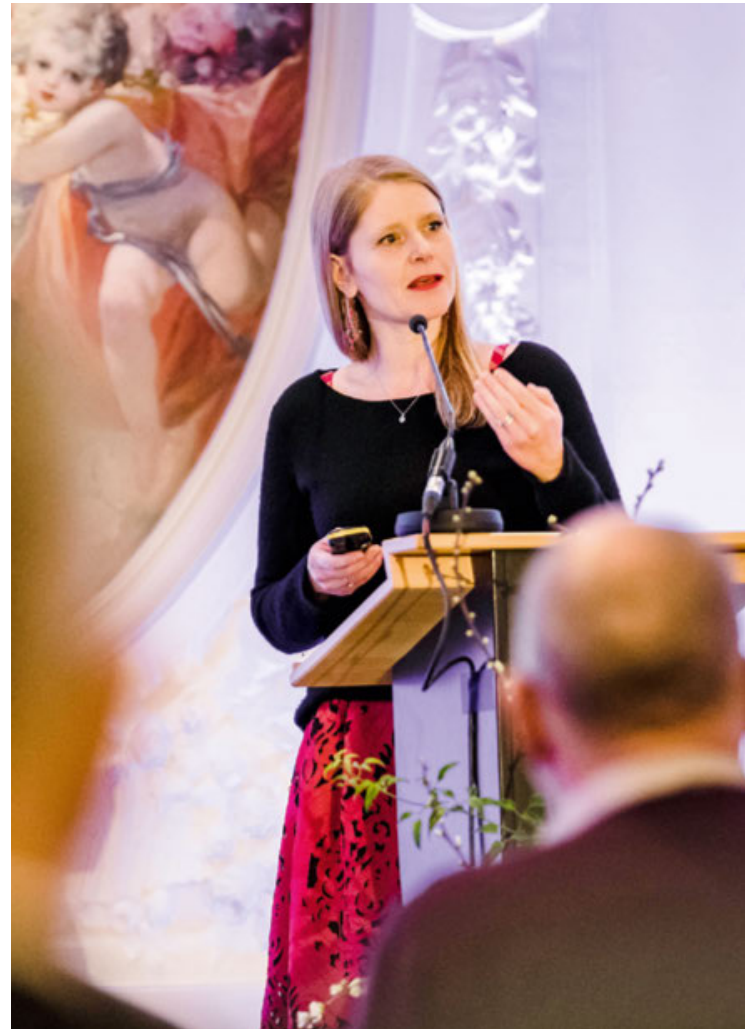

Solange Peters

Auch aus Lausanne kam die Lehr-und Forschungsbeauftragte Carole Clair nach Bern. Das Konzept der Inklusion stamme aus den Sechzigerjahren, die Diskussion über eine gendergerechte Medizin sei also alles andere als neu, aber trotzdem müsse sie immer und immer wieder geführt werden, sagte auch sie. Es sei eine Diskussion, die sich zwischen GeschlechterBlindheit und Geschlechter-Stereotypisierung bewege. Eine Diskussion, bei der es vor allem um ein Bewusstsein gehe. Und dieses müsse unter dem Stichwort "Gender-Teaching» verstärkt in die Aus- und Weiterbildung getragen werden.

\section{Starke Frauen ...}

Zwei Beiträge aus dem Ausland bereicherten die Tagung. Heidi Stensmyren, Vorsitzende der Schwedischen Ärztekammer, machte ihre Präsenz zu ihrer unausgesprochenen Hauptaussage: Schaut hin, auch in der Medizin können es Frauen bis ganz nach oben schaffen - und dabei gelassen bleiben und den Humor nicht verlieren. "Stimmt», sagt die Anästhesistin im persönlichen Gespräch, «aber es brauchte Mut und ich musste mich immer wieder behaupten.» Nach ihrer Wahl 2014 habe sie dann gleich zwei Männer unter sich angestellt, einen Direktor und einen Sekretär. «Und ich merkte, 
dass Männer etwas besser können als wir Frauen: delegieren.» In Schweden übrigens, so berichtete sie, sind die Lohnunterschiede gering, und Ärztinnen und Ärzte arbeiten dort 41,2 Stunden pro Woche.

Vera Regitz-Zagrosek, Professorin für Kardiologie an der Charité in Berlin und «Königin der Geschlechterforschung in der Medizin", wie sie genannt wird, brachte als erste Referentin gleich eine andere Perspektive in diese Tagung: Sie sprach nämlich vor allem über gendergerechte Diagnosen und entsprechend adäquate medizinische Behandlungen. Dies sei eine Frage von Genen und Hormonen, also biologischen Unterschieden, aber auch von soziokulturellen Faktoren wie Rollen und Beziehungen.

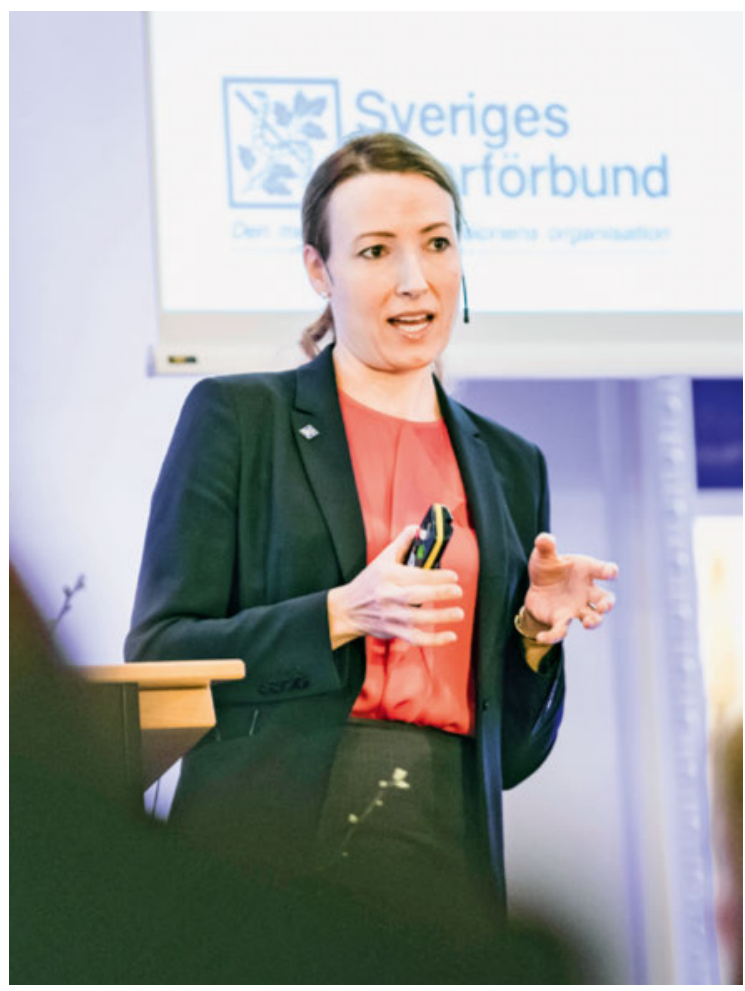

Heidi Stensmyren

So würden Frauen zum Teil ganz andere Schmerzen haben und diese auch anders wahrnehmen als Männer. Oder: So manifestiere sich zum Beispiel eine koronare Herzerkrankung bei den beiden Geschlechtern unterschiedlich. Auf solche Unterschiede sei, wenn Medikamente entwickelt werden, schon bei den Versuchstieren zu achten.

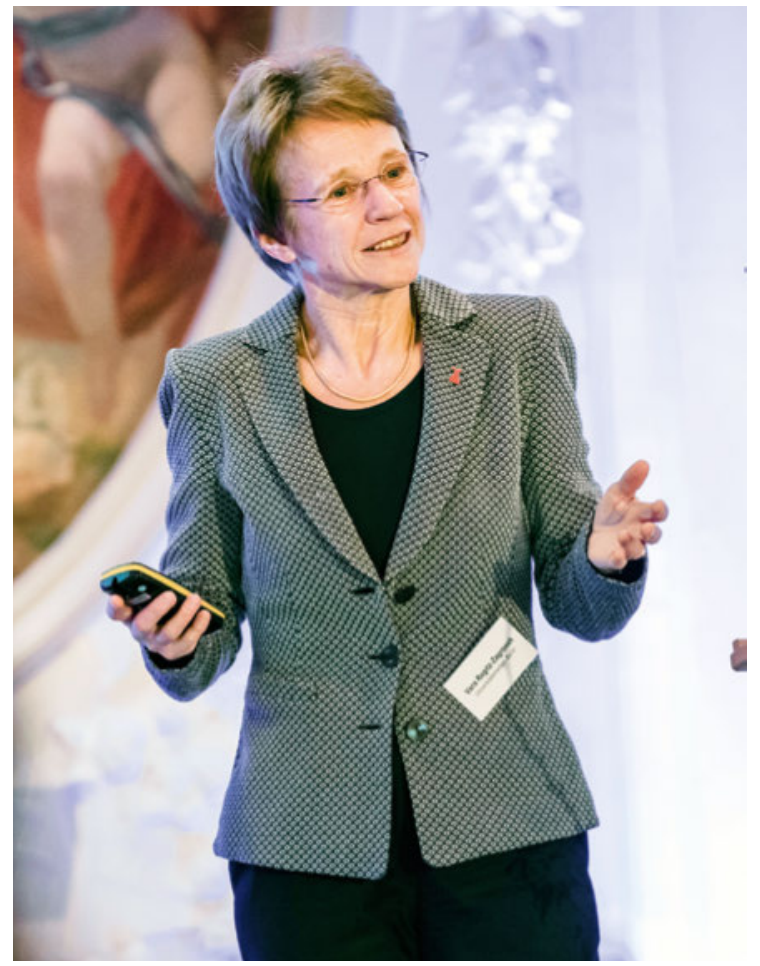

Vera Regitz-Zagrosek

\section{... und schwache Momente}

Das diesjährige, 19. Forumsanté war ein gut organisierter und gut besuchter Anlass. Nebst den erwähnten Frauen referierten dort auch Esther Kraft, Leiterin Abteilung Daten, Demographie und Qualität der FMH und Cornelia Meier, Projektleiterin Grundlagen und Qualität beim Krankenkassenverband santésuisse. Und weil mit Marina Villa auch die Moderation in weiblicher Obhut war, ergab sich bei der grossen - allzu grossen - Diskussionsrunde ein schönes Bild: eine medizinische Tagung mit sieben Frauen auf der Bühne. Zwei Elemente trübten die insgesamt positive Bilanz: die mangelhafte Technik des Hotels Bellevue im ersten Teil, und der Schlusspunkt, den die Journalistin Michèle Binswanger setzte. Ihr überlanger und verworrener Diskurs über den Eroberer Hernán Cortés, den Blutfluss in weiblichen Sexualorganen und eine rosa Pille hatte vor allem den Effekt, die Reihen schnell zu lichten.

Bildnachweis

zVg/Matthias Käser 